\title{
Estimating the tail conditional expectation of Walmart stock data
}

\author{
Hakim Ouadjed ${ }^{1, *}$ and Tawfiq Fawzi Mami ${ }^{2}$ \\ ${ }^{1}$ Faculty of Economics, Business and Management Sciences, Mustapha Stambouli \\ University of Mascara, P.B. 305, 29000 Mascara, Algeria \\ 1 Biomathematics Laboratory, Sidi Bel-Abbes University, Algeria \\ E-mail: 〈oujda.hakim@univ-mascara.dz〉 \\ 2 Science Institute, Belhadj Bouchaib University Center of Ain Témouchent \\ P.B. 284, 46000 Ain Témouchent, Algeria \\ E-mail: 〈mami_math_sba@yahoo.fr $\rangle$
}

\begin{abstract}
Stable distribution, also known as Lévy stable distribution, which is a rich class of heavytailed distributions can capture asymmetry and heavy tails observed in financial data. In this paper, we fit an $\mathrm{AR}(1)$ process with $\alpha$-stable innovations to the logarithms of volumes of Walmart stock traded daily on the New York Stock Exchange and estimate the TCE (Tail Conditional Expectation) risk measure.
\end{abstract}

Keywords: autoregressive process, Lévy stable distribution, risk measure

Received: March 14, 2020; accepted: May 26, 2020; available online: July 07, 2020

DOI: $10.17535 /$ crorr.2020.0008

\section{Stable $\mathrm{AR}(1)$ process}

The heavy-tailed auto-regressive models have various practical applications [7, 12, 18]. Stable distributions are often used to specify the innovations process of auto-regressive processes with infinite variance because of their interesting mathematical properties (heavy tails and asymmetry).

A random variable $X$ has a stable distribution if and only if for every $k$ and any family of independent and identically distributed variables $X_{1}, \ldots, X_{k}$, there exists $a_{k}>0$ and $b_{k}$, two reals, such as:

$$
X_{1}+\ldots+X_{k} \stackrel{D}{=} a_{k} X+b_{k},
$$

where $\stackrel{D}{=}$ denotes equality in distribution. When $b_{k}=0$, we speak of strictly stable distribution. It is shown in [5] that there exists a constant $\alpha, 0<\alpha \leq 2$, such that $a_{k}=k^{1 / \alpha}$ for $k \in \mathbf{N}$.

If $X$ has a stable distribution, then we denoted by $X \sim S(\alpha, \mu, \beta, \sigma)$ and its characteristic function is written as:

$$
\varphi_{X}(t)=\exp \left\{i \mu t-\sigma^{\alpha}|t|^{\alpha}\left(1+i \beta \frac{t}{|t|} w(t, \alpha)\right)\right\}
$$

where

$$
w(t, \alpha)=\left\{\begin{array}{l}
\operatorname{tg}\left(\frac{\alpha \pi}{2}\right) \text { if } \alpha \neq 1 \\
\frac{2}{\pi} \ln |t| \text { if } \alpha=1 .
\end{array}\right.
$$

${ }^{*}$ Corresponding author. 
A stable law is defined by four parameters:

- index of stability $0<\alpha \leq 2$ is the main parameter. It characterizes the distribution tails. If $\alpha$ decreases, the tails are heavy. The case of $\alpha=2$ corresponds to the normal distribution.

- position parameter $\mu \in \mathbf{R}$. It characterizes the law mean when $\alpha>1$.

- asymmetry parameter $-1 \leq \beta \leq 1$. If $\beta=0$, the law is symmetrical about the parameter $\mu$. Moreover, when $\mu=0$ the law is called symmetric $\alpha$-stable law.

- scale parameter $\sigma>0$.

According to [19], the stable laws have the following properties:

1. Let $X_{1}, X_{2}, \ldots$ i.i.d. $\sim S(\alpha, \mu, \beta, \sigma)$ and $c_{j}$ a sequence of real values with $\sum_{j}\left|c_{j}\right|^{\alpha}<\infty$,

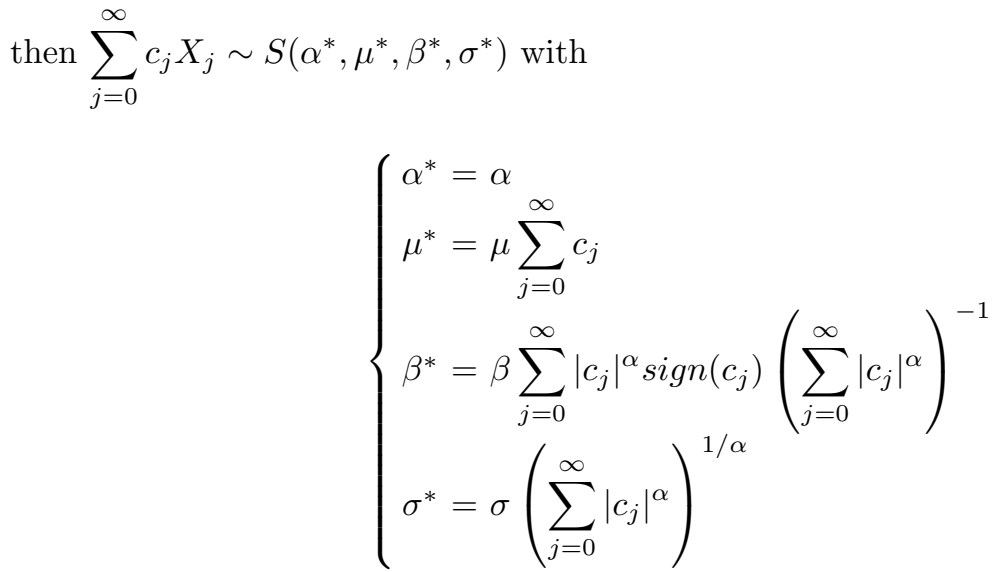

2. If $0<\alpha<2$, the variance of a stable law is infinite and for $0<\alpha<1$, the mean becomes infinite.

3. Let $X \sim S(\alpha, \mu, \beta, \sigma)$, so as $x \rightarrow \infty$, we have

$$
x^{\alpha} \mathbf{P}(X>x) \longrightarrow C_{\alpha} \frac{1+\beta}{2} \sigma^{\alpha}
$$

and

$$
x^{\alpha} \mathbf{P}(X<-x) \longrightarrow C_{\alpha} \frac{1-\beta}{2} \sigma^{\alpha},
$$

where $C_{\alpha}=\frac{2}{\pi} \Gamma(\alpha) \sin \frac{\pi \alpha}{2}$.

If we denote by $G(x):=\mathbf{P}(|X| \leq x)=F_{X}(x)-F_{X}(-x), x>0$, the d.f. of $Z=|X|$, then we have both following conditions:

- The regular variation condition

$$
\lim _{t \rightarrow \infty} \frac{1-G(t x)}{1-G(t)}=x^{-\alpha}, x>0
$$


- The tails balance condition for $0 \leq a \leq 1$

$$
\lim _{x \rightarrow \infty} \frac{1-F(x)}{1-G(x)}=a, \lim _{x \rightarrow \infty} \frac{F(-x)}{1-G(x)}=1-a,
$$

where $a=\frac{1+\beta}{2}$.

The problem of estimating the parameters of a stable distribution is in general hampered by the lack of known closed-form density functions. However, there are numerical methods that have been found useful in practice like the quantile method proposed by [14], the method based on linear regression [11] and the maximum likelihood approach [16].

Let the $\operatorname{AR}(1)$ process:

$$
\begin{aligned}
& X_{t}=\lambda X_{t-1}+\varepsilon_{t}, t=1, \ldots, n \\
& -1<\lambda<1 \\
& \left\{\varepsilon_{t}\right\} \text { i.i.d. } \sim S(\alpha, \mu, \beta, \sigma) \\
& \quad 1<\alpha<2 .
\end{aligned}
$$

The $\mathrm{AR}(1)$ process defined in (8) is strictly stationary and it can be written as:

$$
X_{t}=\sum_{j=0}^{\infty} \lambda^{j} \varepsilon_{t-j}, t=1, \ldots, n
$$

Using (3) and (9) we have $X \sim S\left(\alpha^{*}, \mu^{*}, \beta^{*}, \sigma^{*}\right)$ with

$$
\left\{\begin{array}{l}
\alpha^{*}=\alpha \\
\mu^{*}=\frac{\mu}{1-\lambda} \\
\beta^{*}=\left\{\begin{array}{c}
\beta \quad, 0 \leq \lambda<1 \\
\frac{1-|\lambda|^{\alpha}}{1+|\lambda|^{\alpha}} \beta,-1<\lambda<0
\end{array}\right. \\
\sigma^{*}=\frac{\sigma}{\left[1-|\lambda|^{\alpha}\right]^{1 / \alpha}}
\end{array}\right.
$$

The estimator for the auto-regressive coefficient $\lambda$ is given by the following expression:

$$
\widehat{\lambda}_{n}=\frac{\sum_{i=1}^{n-1}\left(X_{i+1}-\bar{X}_{n}\right)\left(X_{i}-\bar{X}_{n}\right)}{\sum_{i=1}^{n-1}\left(X_{i}-\bar{X}_{n}\right)^{2}},
$$

where $\bar{X}_{n}=\frac{1}{n} \sum_{i=1}^{n} X_{i}$. It has been proven in [2] that this estimator is consistent. 


\section{Tail conditional expectation}

Distortion risk measure $\Pi_{g}$ is a mapping from the set of losses random variables to $R^{+}$defined by

$$
\Pi_{g}(X)=\int_{0}^{\infty} g\left(1-F_{X}(x)\right) d x
$$

where $g:[0,1] \longrightarrow[0,1]$ is a non-decreasing and concave function with $g(0)=0$ and $g(1)=1$. $\Pi_{g}(X)$ was introduced by [3] in terms of Choquet integral and have been extensively been used in finance and insurance [21]. This class of risk measure fulfils all four axioms of a coherent risk measure [1].

The conception of tail distortion risk measure was introduced by [23] as:

$$
\Pi_{g}\left(X \mid X>\operatorname{VaR}_{p}(X)\right)=\int_{0}^{\infty} g_{p}\left(1-F_{X}(x)\right) d x
$$

with

$$
\operatorname{VaR}_{p}(X)=F_{X}^{-1}(p)=\inf \left\{x \in \mathbf{R}: F_{X}(x) \geq p\right\}, 0<p<1
$$

and $g_{p}$ is a distortion function defined by

$$
g_{p}(s)=\left\{\begin{array}{cl}
g\left(\frac{s}{1-p}\right) & \text { if } 0 \leq s \leq 1-p \\
1 & \text { if } 1-p \leq s \leq 1 .
\end{array}\right.
$$

If $g=1$, then we find the tail conditional expectation TCE

$$
\Pi_{g}\left(X \mid X>\operatorname{VaR}_{p}(X)\right)=T C E_{p}(X)=\frac{1}{1-p} \int_{p}^{1} \operatorname{VaR}(X) d s .
$$

Let the order statistic $X_{1, n} \leq X_{2, n} \leq \ldots \leq X_{n, n}$ associated to the sample $\left(X_{1}, X_{2}, \ldots, X_{n}\right)$ of $X$. The empirical estimate of $T C E_{p}(X)$ is:

$$
{\widehat{T C E_{p}(X)}}^{e m p}=\frac{1}{1-p}\left(\frac{1}{n} \sum_{k=[n p]+1}^{n} X_{k, n}+\left(\frac{[n p]}{n}-p\right) X_{[n p], n}\right)
$$

where $[x]$ is the integer part of $x[17]$.

If $X \backsim S(\alpha, 0, \beta, 1), 1<\alpha<2$, [20] have represented TCE as follows:

$$
T C E_{p}(X)=\frac{\alpha}{(1-\alpha)} \frac{\left|V a R_{p}\right|}{\pi(1-p)} \int_{-\bar{\theta}_{0}}^{\pi / 2} g(\theta) \exp \left(-\left|V a R_{p}\right|^{\alpha /(\alpha-1)} v(\theta)\right) d \theta
$$

where

$$
g(\theta)=\frac{\sin \left(\alpha\left(\bar{\theta}_{0}+\theta\right)-2 \theta\right)}{\sin \left(\alpha\left(\bar{\theta}_{0}+\theta\right)\right)}-\frac{\alpha \cos ^{2}(\theta)}{\sin ^{2}\left(\alpha\left(\bar{\theta}_{0}+\theta\right)\right)}
$$

and

$$
v(\theta)=\left(\cos \left(\alpha \bar{\theta}_{0}\right)\right)^{1 /(\alpha-1)}\left[\frac{\cos (\theta)}{\sin \left(\alpha\left(\bar{\theta}_{0}+\theta\right)\right)}\right]^{\alpha /(\alpha-1)} \frac{\cos \left(\alpha\left(\bar{\theta}_{0}+\theta\right)-\theta\right)}{\cos (\theta)},
$$

with

$$
\bar{\theta}_{0}=\frac{1}{\alpha} \arctan \left(\bar{\beta} \tan \left(\frac{\pi \alpha}{2}\right)\right), \bar{\beta}=-\operatorname{sign}\left(\operatorname{VaR}_{p}\right) \beta .
$$


For $Y \backsim S(\alpha, \mu, \beta, \sigma)$, we have $\sigma X+\mu \backsim Y$, then

$$
T C E_{p}(Y)=\sigma T C E_{p}(X)+\mu .
$$

Limit (4) means that $F_{X}$ is in Fréchet maximum domain of attraction [4]. More precisely, for a sample $X_{1}, \ldots, X_{n}$ from the random variable $X \sim S(\alpha, \mu, \beta, \sigma)$, we have

$$
\frac{\max \left(X_{1} \ldots, X_{n}\right)}{F_{X}^{-1}\left(1-n^{-1}\right)} \stackrel{D}{\rightarrow} \Phi_{\alpha}
$$

where $\stackrel{D}{\rightarrow}$ denotes convergence in distribution and

$$
\Phi_{\alpha}(x)= \begin{cases}\exp \left(-x^{-\alpha}\right) & , x>0 \\ 0 & , x \leq 0\end{cases}
$$

The tail index $\alpha$ can be estimated by the Hill estimator [10] defined by

$$
\widehat{\alpha}^{H}=\left[\frac{1}{k} \sum_{i=1}^{k} \log X_{n-i, n}-\log X_{n-k+1, n}\right]^{-1}
$$

where $k=k_{n}$ is an intermediate sequence such that $k \rightarrow \infty, k / n \rightarrow 0, n \rightarrow \infty$. form

The semi-parametric estimator of a high quantile $(p \rightarrow 1)$ proposed in [22] has the following

$$
\widehat{\operatorname{VaR}}_{p}^{H}=X_{n-k, n}\left(\frac{k}{n(1-p)}\right)^{1 / \widehat{\alpha}^{H}} .
$$

It is known (e.g., [23]) that, for $p \rightarrow 1$ and $\alpha>1$ we have

$$
T C E_{p}=\frac{\alpha}{\alpha-1} V a R_{p}
$$

Then we obtain the following estimator

$$
\widehat{T C E}_{p}^{H}=\frac{\widehat{\alpha}^{H}}{\widehat{\alpha}^{H}-1} X_{n-k, n}\left(\frac{k}{n(1-p)}\right)^{1 / \widehat{\alpha}^{H}} .
$$

\section{Application}

Our study is carried out on the natural logarithms of the volumes of Walmart stock traded daily on the New York Stock Exchange (time-series $X_{t}$ ) during the period from November 19, 2003 to January 4, 2005. The observations are shown on the Figure 1 and their empirical density is plotted on the Figure 2. The data, were taken from the public source https://finance. yahoo.com. 


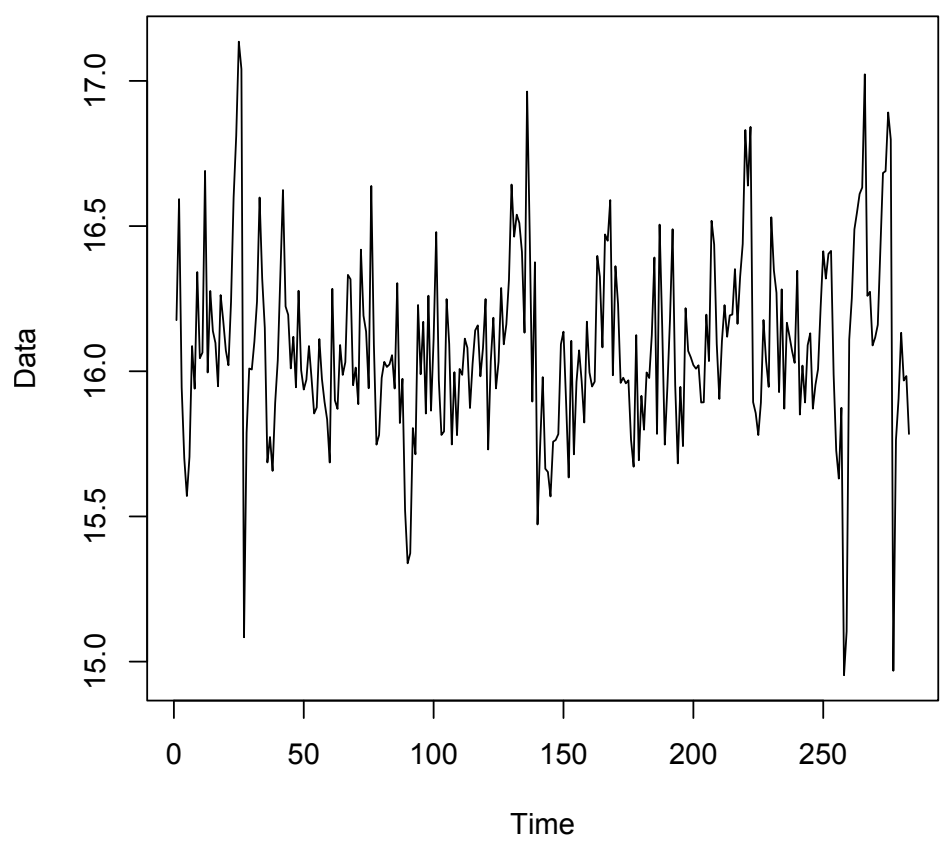

Figure 1: Daily logarithms of the volumes of Walmart stock

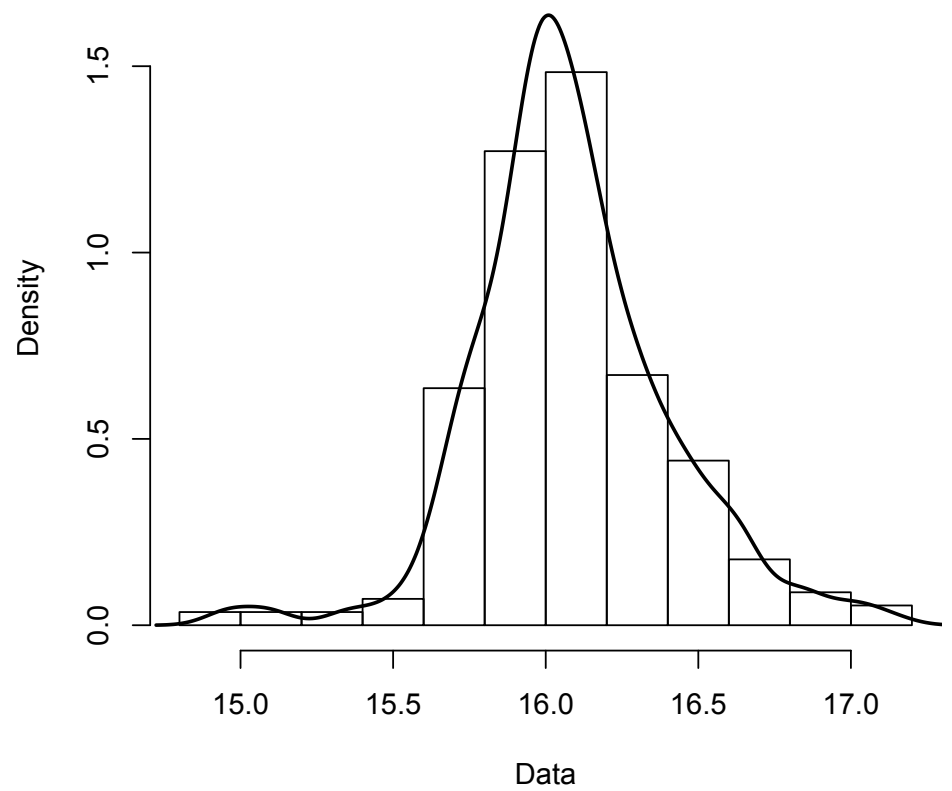

Figure 2: Empirical density of time-series $X_{t}$

To verify the stationarity of the data, we perform a Phillips-Perron test, we get a $p-$ value of 0.01 , so the data are stationary. The data exhibit a high excess kurtosis $4.593>3$, indicating that the observations are not normally distributed. The $p$-value of the Kolmogorov-Smirnov normality test is $4.7 \times 10^{-6}$, thus confirming the rejection of the assumption that the data would normally be distributed. 
On the Figure 3, since the ACF decreases gradually and the partial ACF cut off after the first lag, it seems that the data follow an $\mathrm{AR}(1)$ process.
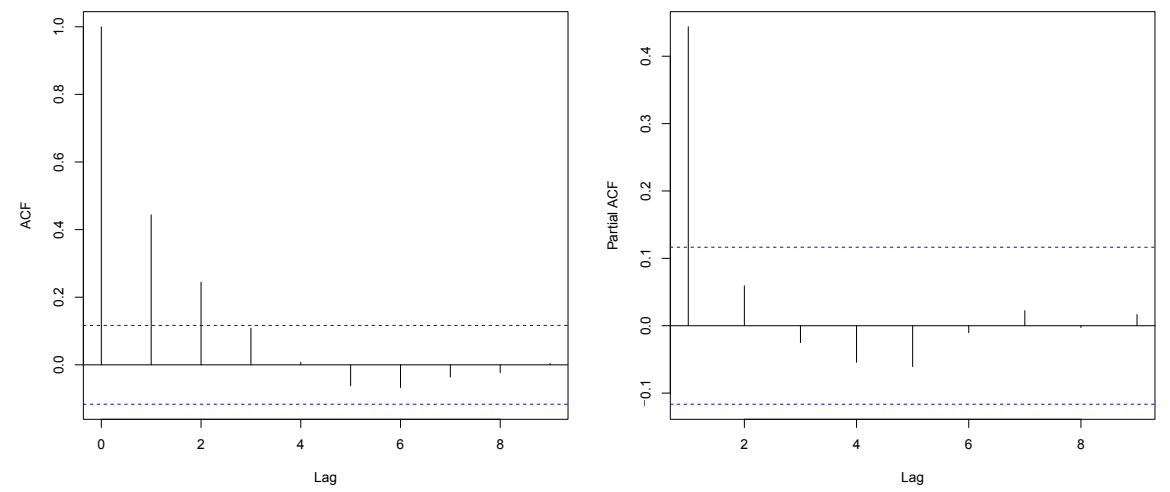

Figure 3: $A C F$ and PACF of time-series $X_{t}$

Moreover, we use the AICC (Akaike's Information Criteria Corrected) and BIC (Bayesian Information Criterion) for all $A R M A(p, q)$ models with $p+q \leq 2$ as given in the Table 1 .

\begin{tabular}{|c|c|c|}
\hline$A R M A(p, q)$ & $A I C C$ & $B I C$ \\
\hline \hline $\operatorname{AR}(1)$ & 96.67423 & 100.3054 \\
$\operatorname{AR}(2)$ & 97.68286 & 104.9309 \\
$\mathrm{MA}(1)$ & 110.6942 & 114.3254 \\
$\mathrm{MA}(2)$ & 101.2229 & 108.4709 \\
$\mathrm{ARMA}(1,1)$ & 97.82286 & 105.0709 \\
\hline
\end{tabular}

Table 1: AICC and BIC criteria's for different ARMA $(p, q)$ models

From this, it is obvious that the best model for $X_{t}$ with respect to both criteria was an $\operatorname{AR}(1)$ defined by

$$
X_{t}=\lambda X_{t-1}+\varepsilon_{t}, t=1, \ldots, 283
$$

with $X_{0}=0$ and $\left\{\varepsilon_{t}\right\}$ i.i.d. whose distribution we will specify later.

The estimator provided by (11) of the coefficient $\lambda$ is $\widehat{\lambda}=0.4437255$. Statistical analysis of the residuals $\widehat{\varepsilon}_{t}=X_{t}-\widehat{\lambda} X_{t-1}, 2 \leq t \leq 283$, leads to the following results:

1. The empirical ACF and PACF of the residuals on the Figure 4 shows the non-significance of auto-correlation coefficients.
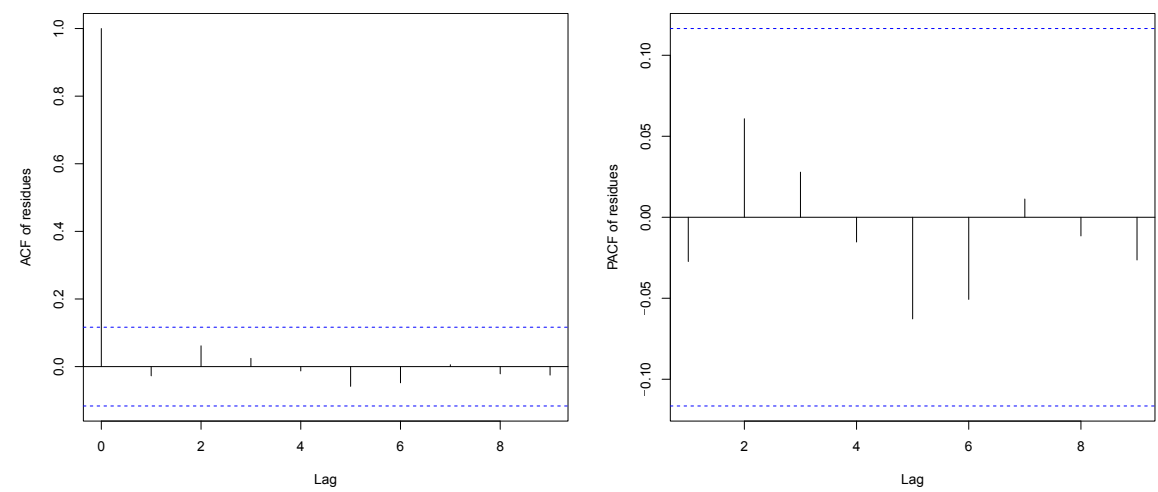

Figure 4: The empirical $A C F$ and PACF of the residuals $\widehat{\varepsilon}_{t}$ 
2. Empirical density function of residuals on the left of the Figure 5 shows some asymmetry and the kurtosis of $6.941248>3$ indicates a heavy tail of distribution, which is confirmed by the deviation at the extremes of the normal Q-Q plot (Figure 5 on the right).
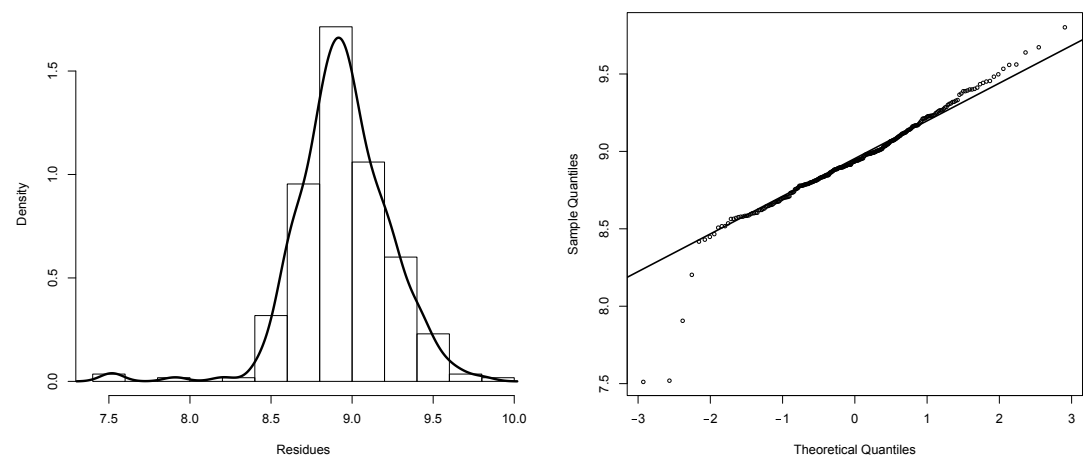

Figure 5: Empirical density of $\widehat{\varepsilon}_{t}$ (left) and the normal $Q-Q$ plot (right)

The phenomena of heavy tails and excess kurtosis of the residues $\widehat{\varepsilon}_{t}$ suggest that a stable distribution $S(\alpha, \mu, \beta, \sigma)$ would be an appropriate model for $\widehat{\varepsilon}_{t}$. To estimate the parameters $\alpha, \mu, \beta$ and $\sigma$ we use the method of McCulloch [14]. The results are summarized in Table 2.

\begin{tabular}{|c|c|c|c|}
\hline$\widehat{\alpha}$ & $\widehat{\mu}$ & $\widehat{\beta}$ & $\widehat{\sigma}$ \\
\hline \hline 1.834 & 8.9203869 & 0.95 & 0.1701674 \\
\hline
\end{tabular}

Table 2: Stable parameters of residuals

To estimate the parameters of the $\operatorname{AR}(1)$ process defined in (25) we use the equations (10). The results are given in Table 3.

\begin{tabular}{|c|c|c|c|}
\hline$\widehat{\alpha^{*}}$ & $\widehat{\mu^{*}}$ & $\widehat{\beta^{*}}$ & $\widehat{\sigma^{*}}$ \\
\hline \hline 1.834 & 16.03594 & 0.95 & 0.1955845 \\
\hline
\end{tabular}

Table 3: Stable parameters of $A R(1)$

In the Figure 6 the plots of the empirical density of the data and the estimated stable density $S\left(\widehat{\alpha^{*}}, \widehat{\mu^{*}}, \widehat{\beta^{*}}, \widehat{\sigma^{*}}\right)$ show a good fit.

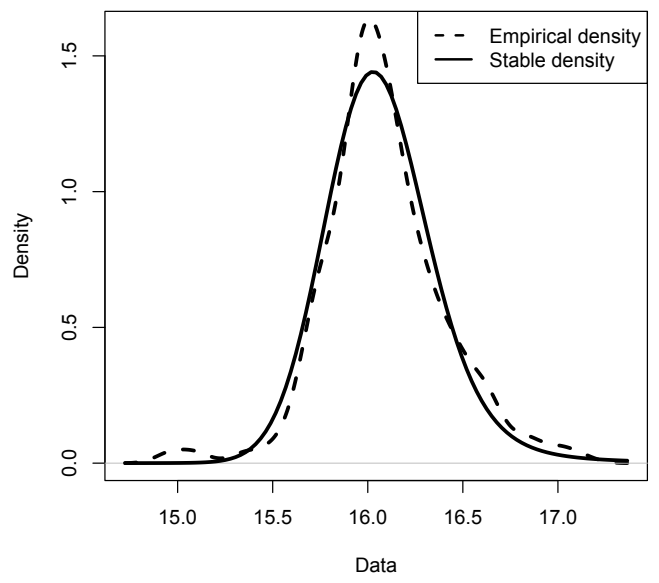

Figure 6: The goodness of fit 
To confirm this, the $p$-value $=0.6161$ of the Kolmogorov-Smirnov test implies that for our data there a better fit of the stable model around the center of the distribution while the $p$-value $=0.3176$ of the Anderson-Darling test implies a better fit in the tails at the significance level $5 \%$. The $P-P$ plot on the Figure 7 is linear, also confirming the quality of fit for the stable distribution.

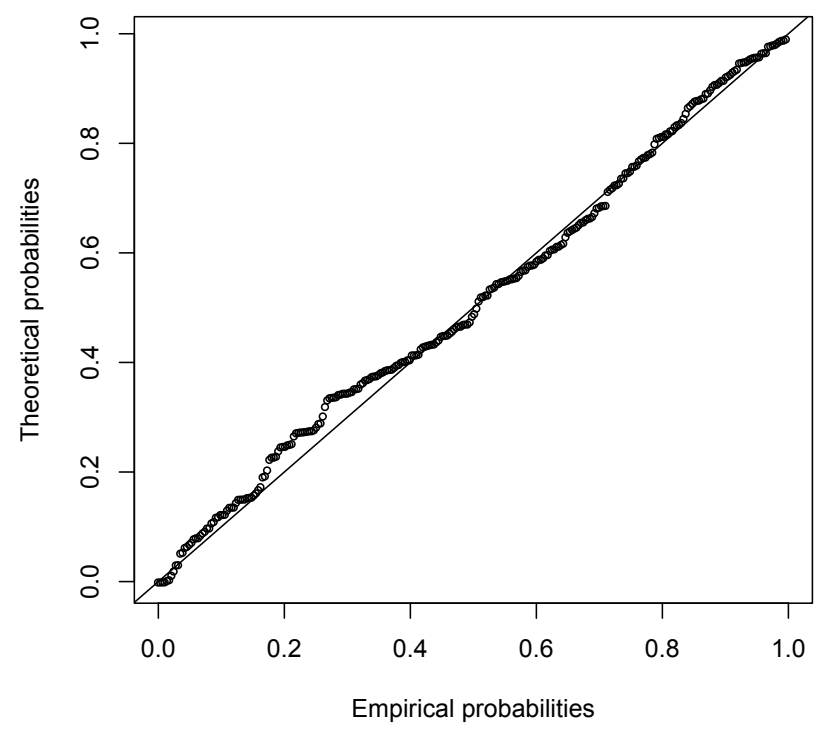

Figure 7: The P-P plot

From (18) we calculate the estimator $\widehat{T C E}_{X_{t}}^{1}$ using the parameters $\widehat{\alpha^{*}}, \widehat{\mu^{*}}, \widehat{\beta^{*}}$ and $\widehat{\sigma^{*}}$.

We adjust a stable law to the data $X_{t}$ without passing by the residuals using the McCulloch estimators (Table 4).

\begin{tabular}{|c|c|c|c|}
\hline$\widehat{\alpha}_{X_{t}}$ & $\widehat{\mu}_{X_{t}}$ & $\widehat{\beta}_{X_{t}}$ & $\widehat{\sigma}_{X_{t}}$ \\
\hline \hline 1.678 & 16.0172717 & 0.821 & 0.1788372 \\
\hline
\end{tabular}

Table 4: Stable parameters obtained by McCulloch estimators

Then, using stable parameters from Table 4, we calculate $\widehat{T C E}_{X_{t}}^{2}$ from (18). For comparison, we calculate the empirical $\widehat{T C E}^{e m p}$ of the real data using (17) which are presented in Table 5.

\begin{tabular}{|c|c|c|}
\hline$p$ & 0.90 & 0.95 \\
\hline \hline$\widehat{T C E}^{e m p}$ & 16.70186 & 16.83251 \\
$\widehat{T C E}_{X_{t}}^{1}$ & 16.66029 & 16.81588 \\
$\widehat{T C E}_{X_{t}}^{2}$ & 16.75967 & 17.00363 \\
\hline
\end{tabular}

Table 5: Estimation of TCE at 90\% and 95\% confidence levels

To apply the Hill estimator for data coming from stable distribution, [6] proposed to center the data by subtracting the median. In the Figure 8 we have plotted $\left(k, \widehat{\alpha}^{H}\right)$ for the data $Y_{t}=X_{t}-\operatorname{median}\left(X_{t}\right)$. 


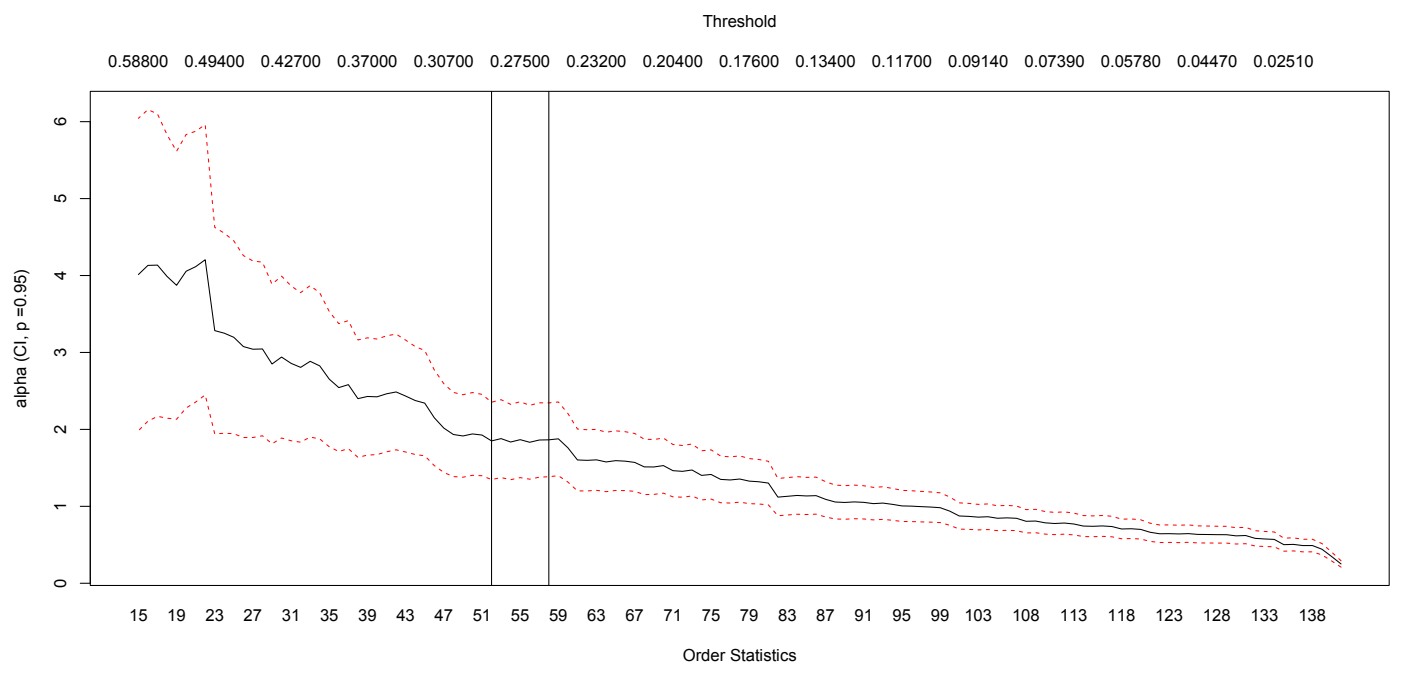

Figure 8: Hill estimator for $Y_{t}$

We see that there is a region of stability between $k=52$ and $k=58$, so we compute $\widehat{\alpha}^{H}$ for each value of this region and calculate $\widehat{T C E}_{Y_{t}}^{H}$ using (24), then we deduce $\widehat{T C E}_{X_{t}}^{H}$ from the following equation:

$$
\widehat{T C E}_{X_{t}}^{H}=\widehat{T C E}_{Y_{t}}^{H}+\operatorname{median}\left(X_{t}\right)
$$

and obtain results in the Table 6.

\begin{tabular}{|c|c|c|c|}
\cline { 3 - 4 } \multicolumn{2}{c|}{} & \multicolumn{2}{c|}{$\widehat{T C E}_{X_{t}}^{H}$} \\
\hline$k$ & $\widehat{\alpha}^{H}$ & $p=0.90$ & $p=0.95$ \\
\hline \hline 52 & 1.844519 & 16.91662 & 17.31423 \\
53 & 1.801733 & 16.93763 & 17.35649 \\
54 & 1.832314 & 16.92239 & 17.32583 \\
55 & 1.799902 & 16.93892 & 17.35889 \\
56 & 1.829332 & 16.92386 & 17.32878 \\
57 & 1.832247 & 16.92248 & 17.32599 \\
58 & 1.845455 & 16.91603 & 17.31314 \\
\hline
\end{tabular}

Table 6: $\widehat{T C E}_{X_{t}}^{H}$ for $52 \leq k \leq 58$.

From the Tables 5-6 we remark following:

1. The estimators of a stable $\mathrm{AR}(1)$ via the residuals estimators give better results of $\widehat{T C E}$ than the estimators applied directly to the data. [13] have shown by simulations on synthetic samples that the estimates of the distribution parameters of the $\alpha$-stable $\operatorname{AR}(1)$ process are better via the residuals.

2. The values of $\widehat{T C E}^{H}$ are farther from the values of $\widehat{T C E}^{e m p}$ because [15] shows that the Hill estimator performs poorly on stable data when $1<\alpha<2$ leads to overestimates of $\alpha$ and thus overestimates the $T C E^{H}$. 


\section{Conclusion}

In this paper, we adjusted a stable AR(1) model to the logarithms of the volumes of Walmart stock data and estimated the coherent risk measure TCE taking into account the dependence structure that exists in the model by estimating the parameters of the stable AR(1) via the residuals. Results are similar to [9] assuming the hypothesis of independence of the data involved in the modeling. The continuous evolution of risks in insurance and finance leads to reflections aimed at relaxing this hypothesis.

There are many possibilities for defining dependency, e.g. [8] estimated the TCE risk measure using the extremal index and the POT method.

Among the many possible mathematical tools to take into account such dependencies, we find the copulas which allow the introduction and characterization of a very flexible form of dependence between different random variables. It would be interesting to mix the concept of copula with the auto-regressive processes to describe another form of dependence in the multivariate case. These are topics for future research.

\section{Acknowledgements}

This work was partially supported by the Ministry of Higher Education and Scientific Research MESRS - Director General for Scientific Research and Technological Development DGRSDT, PRFU: COOLO3UN220120190001.

The authors would like to thank the reviewers for their valuable comments and suggestions that greatly improved the presentation of this research.

\section{References}

[1] Artzner, P., Delbaen, F., Eber, J-M. and Heath, D. (1999). Coherent measures of risk. Mathematical Finance, 9(3), 203-228. doi: 10.1111/1467-9965.00068

[2] Davis, R. and Resnick, S. (1986). Limit theory for the sample covariance and correlation functions of moving averages. The Annals of Statistics, 14(2), 533-558. doi: 10.1214/aos/1176349937

[3] Denneberg, D. (1994). Non-additive measure and integral. Dordrecht: Springer doi: 10.1007/97894-017-2434-0

[4] Embrechts, P., Klüppelberg, C. and Mikosch, T. (1997). Modelling extremal events for insurance and finance. Berlin: Sringer. doi: 10.1007/978-3-642-33483-2

[5] Feller, W. (2008). An introduction to probability theory and its applications-Volume 2 (2nd edition). John Wiley and Sons.

[6] Fofack, H. and Nolan, J. P. (1999). Tail behavior, modes and other characteristics of stable distributions. Extremes, 2(1), 39-58. doi: 10.1023/A:1009908026279

[7] Gallagher, C. M. (2001). A method for fitting stable autoregressive models using the autocovariation function. Statistics and Probability Letters, 53(4), 381-390. doi: 10.1016/s01677152(01)00041-4

[8] Hakim, O. (2018). POT approach for estimation of extreme risk measures of EUR/USD returns. Statistics, Optimization and Information Computing, 6(2), 240-247. doi: 10.19139/soic.v6i2.395

[9] Hakim, O. (2019). Statistical modelling of the EUR/DZD returns with infinite variance distribution. Pakistan Journal of Statistics and Operation Research, 15(2), 451-460. doi: 10.18187/pjsor.v15i2.2654

[10] Hill, B. M. (1975). A simple general approach to inference about the tail of a distribution, The Annals of Statistics, 3(5), 1136-1174. doi: 10.1214/aos/1176343247

[11] Koutrouvelis, I. A. (1980). Regression-type estimation of the parameters of stable laws. Journal of the American Statistical Association, 75(372), 918-928. doi: 10.1080/01621459.1980.10477573

[12] Ling, S. (2005). Self-weighted least absolute deviation estimation for infinite variance autoregressive models. Journal of the Royal Statistical Society: Series B (Statistical Methodology), 67(3), 381-393. doi: 10.1111/j.1467-9868.2005.00507.x 
[13] Mami, T. and Yousfate, A. (2013). On parameters estimation of stationary AR(1) with nonzero mean alpha-stable innovations in the case $\alpha \in] 1,2]$. Acta Universitatis Apulensis, 35, 37-64. http: //auajournal.uab.ro/upload/59_941_04.pdf

[14] McCulloch, J. H. (1986). Simple consistent estimators of stable distribution parameters. Communications in Statistics-Simulation and Computation, 15(4), 1109-1136. doi: $10.1080 / 03610918608812563$

[15] McCulloch, J. H. (1997). Measuring tail thickness to estimate the stable index $\alpha$ : A critique. Journal of Business and Economic Statistics, 15(1), 74-81. doi: 10.1080/07350015.1997.10524689

[16] Nolan, J. P. (2001). Maximum likelihood estimation and diagnostics for stable distributions. In Barndorff-Nielsen O. E., Resnick S. I. and Mikosch T. (Eds.), Lévy Processes (pp. 379-400). Boston: Birkhäuser. doi: 10.1007/978-1-4612-0197-7_17

[17] Rachev, S. T., Stoyanov, S. W. and Fabozzi, F. J. (2008). Advanced stochastic models, risk assessment and portfolio optimization: the ideal risk, uncertainty and performance measures. John Wiley and Sons.

[18] Resnick, S. I. (1997). Heavy tail modeling and teletraffic data: special invited paper. The Annals of Statistics, 25(5), 1805-1869. doi: 10.1214/aos/1069362376

[19] Samorodnitsky, G. and Taqqu, M. (1994). Stable non-gaussian random processes: stochastic models with infinite variance. New York: Routledge. doi: 10.1201/9780203738818

[20] Stoyanov, S. V., Samorodnitsky, G., Rachev, S. and Ortobelli Lozza, S. (2006). Computing the portfolio conditional Value-at-risk in the alpha-stable case. Probability and Mathematical Statistics, 26(1), 1-22. https://ssrn. com/abstract $=1729002$

[21] Wang, S. (1996). Premium calculation by transforming the layer premium density. ASTIN Bulletin: The Journal of the IAA, 26(1), 71-92. doi: 10.2143/ast.26.1.563234

[22] Weissman, I. (1978). Estimation of parameters and large quantiles based on the $\mathrm{k}$ largest observations. Journal of the American Statistical Association, 73(364), 812-815. doi: 10.1080/01621459.1978.10480104

[23] Zhu, L. and Li, H. (2012). Tail distortion risk and its asymptotic analysis. Insurance: Mathematics and Economics, 51(1), 115-121. doi: 10.1016/j.insmatheco.2012.03.010 